\title{
Throughput Analysis and Optimization of Multi-layer FFR-aided OFDMA Networks
}

\author{
Jan García-Morales, Guillem Femenias, Felip Riera-Palou, and John S. Thompson. \\ Mobile Communications Group - University of the Balearic Islands. \\ Institute for Digital Communications - The University of Edinburgh. \\ \{jan.garcia,guillem.femenias,felip.riera\}@uib.es, john.thompson@ed.ac.uk
}

\begin{abstract}
In OFDMA networks, the use of universal frequency reuse plans improves cell capacity but causes very high levels of inter-cell interference (ICI), particularly affecting users located in the cell-edge regions. In order to mitigate ICI while achieving high spectral efficiencies, fractional frequency reuse (FFR) shows a good tradeoff between celledge throughput and overall cell spectral efficiency. Recently, multi-layer FFR-aided OFDMA-based designs, splitting the cell into inner, middle and outer layers have been proposed and studied with the aim of increasing the spectrum utilization and improving the user fairness throughout the cell. This paper presents an analytical framework allowing the performance evaluation and optimization of multi-layer FFR designs in OFDMA-based networks. Tractable mathematical expressions of the average cell throughput as well as the layer spectral efficiency have been derived for both proportional fair (PF) and round robin (RR) scheduling policies.
\end{abstract}

Keywords-OFDMA cellular networks, multi-layer FFR, spectral efficiency, throughput, optimization.

\section{INTRODUCTION}

Orthogonal frequency division multiple access (OFDMA) is one of the most prominent air-interfaces in modern cellular standards [1]. Owing to the orthogonality among subcarriers, OFDMA makes the intra-cell interference negligible. However, inter-cell interference (ICI) remains an issue due to the use of aggressive high spectral efficiency universal frequency reuse plans where all cells use the same set of frequency subbands (reuse-1). In this setup, ICI critically affects the user mobile stations (MSs) located in the edge of the cells because the serving base station (BS) and the interfering ones are at similar distances. In contrast, the well-known reuse-3 scheme decreases ICI but sacrificing spectral efficiency. With the aim of mitigating ICI experienced by the cell-edge users while still achieving high spectral efficiencies, multiple ICI control (ICIC) strategies have been proposed [2], among which, static fractional frequency reuse (FFR) and all its variants show a good tradeoff among cell-edge throughput enhancement, provision of high spectral efficiency and implementation complexity [3].
The FFR scheme divides the cell into two layers, the inner and the outer one (also known as cell-center and cell-edge regions). In FFR-based cellular systems, a low frequency reuse factor is used for the cell-inner MSs (typically reuse-1), less affected by co-channel interference, and a larger frequency reuse factor is selected for the cell-outer MSs (e.g., reuse-3). However, traditional two-layer FFR has some drawbacks: (i) when the inner layer is large, the MSs located in the edge of the inner layer suffer from high levels of ICI, (ii) when the outer layer becomes large, the spectrum utilization becomes low and the spectral efficiency drops. In an attempt to reconcile these two conflicting situations, FFR schemes with more than 2 layers have been recently proposed [4]. The main idea of the multi-layer FFR scheme is to increase the spectrum utilization, enhance the average cell throughput and improve the MS fairness throughout the cell by incorporating middle layers in between the inner and outer ones.

Regardless of the particular ICIC technique in use, spectral efficiency can be significantly enhanced by using channel-aware schedulers that allocate, on a slot-by-slot basis, each subcarrier to a user with favourable channel conditions (i.e., a user experiencing a high signalto-interference-plus-noise ratio (SINR)), thus exploiting multiuser diversity. Remarkably, the proportional fair (PF) scheduler has been shown to provide a good tradeoff between spectral efficiency and fairness [5]. Then, ICI can be decreased using a PF scheduler in combination with FFR schemes, while at the same time the possibility of a MS with a very bad link suffering from long periods of starvation can be drastically reduced.

The analytical performance evaluation of FFR-aided OFDMA-based cellular networks has been tackled using Poisson Point Processes (PPPs) for modeling the location of the BSs [6], [7]. This approach allows the characterization of the system performance by spatially averaging over all possible network realizations, but precludes from accurately analyzing the performance of a given cell, 
a metric of particular importance to network designers that, provided a planned set of BS locations along with traffic load conditions, may be interested in calculating the performance obtained within a specific region in the coverage area of the network. Fan Jin et al. [8] studied an FFR-aided twin-tier OFDMA network where stochastic geometry was used to characterize the random distribution of femtocells, and the macrocells were overlaid on top of the femtocells following a regular tessellation. However, the analytical framework was limited to resource allocation schemes based on the round robin scheduling policy. Similar approaches, lacking the consideration of scheduling policies and small scale fading, were also proposed by Assaad in [9] and Najjar et al. in [10] to optimize FFR-based parameters in a single-tier network. These limitations were overcome in part by $\mathrm{Xu}$ et al. in [11] and Garcia et al. in [12] (see also [13]), but only taking into account the use of opportunistic maximum SINR (MSINR) schedulers.

In contrast to the above background work, following studies have considered the use of the multi-layer FFR scheme to control the ICI. Xie and Walke [14] proposed a three-layer FFR scheme using reuse-1 and low power for inner layer, reuse- 3 and moderate power for middle layer, and reuse-9 and high power for outer layer. A theoretical analysis of a series of reuse partitioning approaches was carried out in this paper using mathematically tractable expressions, but with the only consideration of the pathloss effect and thus precluding any attempt to analyze the system performance under the use of channelaware schedulers. Ghaffar and Knopp [15] proposed a three-layer scheme that divided the whole spectrum into four subbands. They used reuse- 1 for the inner layer and reuse-3/2 for the middle and outer layers. The use of this approach provided a reduction of power consumption at the BSs leading to an improvement of the average spectral efficiency but at the cost of increasing the ICI. A multi-layer soft frequency reuse (SFR) scheme was proposed by Yang in [16] with different power levels for each layer. Using this approach allowed the achievement of a better interference pattern than that obtained using a two-layer SFR, thus improving the overall spectral efficiency. In [15] and [16], the average cell and layer spectral efficiencies were formulated, but the authors did not provide neither closed-form solutions nor mathematically tractable expressions and consequently, only results obtained through Monte-Carlo simulations were presented. Particularly interesting is the work of Wang et al. in [4], where a tractable multi-layer FFR model was proposed. Moreover, optimal designs and closed-form expressions of the average spatial capacities of certain typical regions of a cell were derived. One of the main conclusions of this work was that multi-layer schemes can provide better average spatial capacity and fairness than the traditional two-layer scheme. The main limitation of this work, however, was the use of rather unrealistic assumptions such as neglecting the small scale fading effects and, consequently, limiting the proposed analytical framework to resource allocation schemes based on the round robin scheduling policy.

In this paper, a novel approach for a multi-layer FFRaided downlink OFDMA-based multi-cellular network is introduced, studied and compared. To this end, an analytical framework is presented allowing the performance evaluation of both two-layer FFR and multi-layer FFR using a PF or a RR scheduling policy. The main contributions of this paper can be summarized as follows:

- Based on the statistical channel characterization and a unified cell throughput approach, an analytical framework allowing the evaluation of the impact that any of the FFR layers may produce on the cell throughput is provided.

- Tractable mathematical expressions of the average cell throughput as well as the layer capacity have been derived for both proportional fair (PF) and round robin (RR) scheduling policies.

- The worst MSs, typically located at the edge of each layer, are considered in the analysis aiming at determining the size of the fractional frequency schemerelated spatial and frequency partitions guaranteeing proper QoS and fairness levels throughout the cell coverage.

It is worth stressing at this point that the proposed analytical framework also opens the door to the theoretical spectral efficiency evaluation and optimization of OFDMA-based cellular networks using more sophisticated ICIC techniques such as adaptive frequency reuse or network MIMO, as well as to the assessment of cellular heterogeneous networks.

\section{CELLULAR NETWORK MODEL}

Let us consider the downlink of an OFDMA-based cellular system where a set of BSs are assumed to be deployed following a conscious planning and thus, are regularly arranged over the whole coverage area. This cellular environment can be safely modeled as a regular tessellation of hexagonally-shaped coverage areas, as shown in Figs. 1 and 2 , with the BSs located at the centre of the hexagons ${ }^{1}$. For the sake of analytical tractability, the central cell, covered by BS 0 , which will be referred to as the tagged BS, will be approximated by a circle whose area is the same as the hexagonal one. That is, assuming that the side of the regular hexagon is $R_{h}$, the radius of the circular cell is $R=R_{h} \sqrt{3 \sqrt{3} /(2 \pi)}$, and the total cell coverage area is $A_{r}^{T}=\pi\left(R^{2}-R_{0}^{2}\right)$, where $R_{0}$ is the minimum distance of a MS from its serving BS.

The locations of the MSs at a given time instant are assumed to form a stationary PPP of normalized intensity $\lambda$ (measured in MSs per area unit). A consequence of this assumption is that the probability distribution of the number $M_{\mathcal{S}}$ of MSs falling within any spatial region $\mathcal{S}$ of area $A_{r}^{\mathcal{S}}$ follows a Poisson distribution, thus implying

$$
\mathbb{P}\left\{M_{\mathcal{S}}=k\right\}=\frac{\left(\lambda A_{r}^{\mathcal{S}}\right)^{k} e^{-\lambda A_{r}^{\mathcal{S}}}}{k !} .
$$

${ }^{1}$ Omnidirectional antenna BSs are assumed in this paper. In future work this will be extended to consider the use of sectorization. 


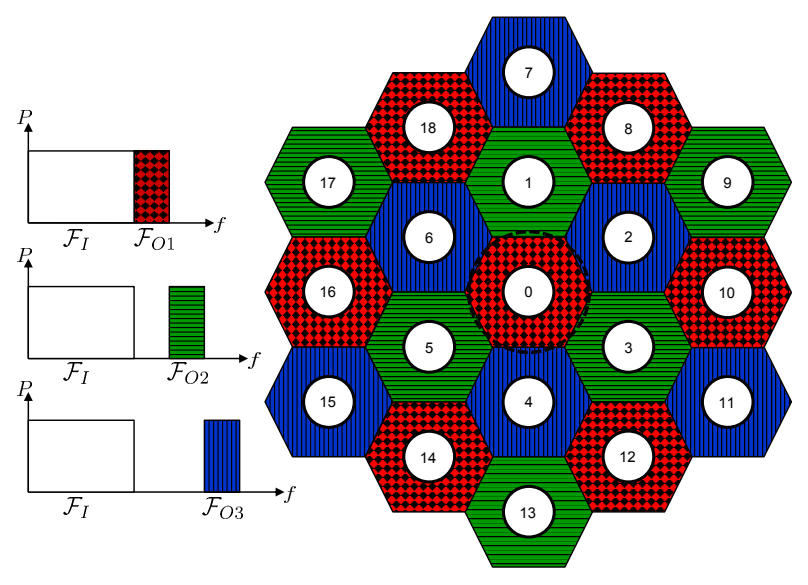

Fig. 1: Schematic representation of the two-layer FFRaided OFDMA-based cellular network.

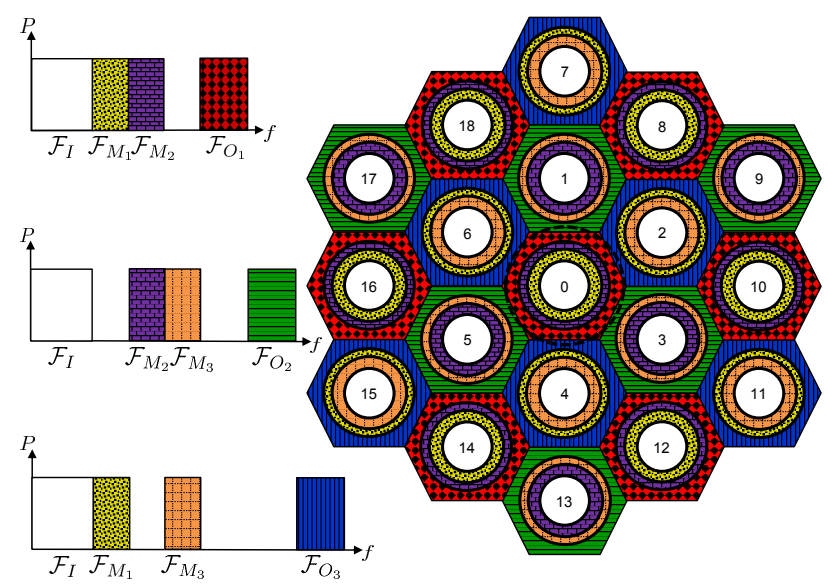

Fig. 2: Schematic representation of a multi-layer FFRaided OFDMA-based cellular network.

\section{A. Two-layer FFR network layout}

In order to control the ICI, MSs are classified according to the received average SINR as either cell-inner MSs, when the received average SINR is above a given threshold, or cell-outer MSs, when it is below the threshold. A two-layer FFR scheme is then applied by allocating non-overlapping resources (subcarriers) to cell-inner and cell-outer MSs, while employing a frequency reuse factor equal to one (reuse-1) for the cell-inner MSs and a higher frequency reuse factor for the cell-outer MSs that, is assumed to be 3 in this paper (reuse-3). For analytical tractability, inner and outer regions (or layers) will be separated by a circumference of radius $R_{t h}$ (threshold distance).

The total system bandwidth is exploited by means of a set $\mathcal{F}_{T}$ of $N_{T}$ orthogonal subcarriers with a bandwidth $\Delta f$ small enough to assume that all subcarriers experience frequency flat fading. The set $\mathcal{F}_{T}$ is split into a set $\mathcal{F}_{I}$ of subcarriers allocated to the inner layer and a set $\mathcal{F}_{O}=$ $\mathcal{F}_{T} \backslash \mathcal{F}_{I}$ of subcarriers allocated to outer layers. The set $\mathcal{F}_{O}$ is further split into three equal parts, namely $\mathcal{F}_{O 1}$, $\mathcal{F}_{\mathrm{O} 2}$ and $\mathcal{F}_{\mathrm{O} 3}$, which are allocated to outer-cell MSs in such a way that adjacent cells will operate on different sets of subcarriers, as shown in Fig. 1. Note that, denoting by $N_{I}$ and $N_{O}$ the number of subcarriers allocated to the inner layer and each of the outer layers, respectively, it holds that $N_{T}=N_{I}+3 N_{O}$.

\section{B. Multi-layer FFR network layout}

When multi-layer FFR scheme is applied, a middle layer is inserted between the inner and the outer ones. This is necessary because neither the inner layers nor the outer layers should be large as explained in Section I. The reuse factor of inner layers should be small (e.g., reuse-1) to keep a relatively high spectrum utilization. Meanwhile, the reuse factor of outer layers should be large (e.g., reuse3 ) in order to avoid high levels of ICI affecting the MSs located far from the BS. Wang et al. in [4] divide the middle layer into two sublayers, i.e., middle1 and middle2, with reuse factor 3/2, as shown in Fig. 2, that is a feasible and practical choice for the design of a multi-layer FFR scheme, and also preferred from a performance point of view. The inner and outer layers are designed in the same way as the traditional FFR scheme. Again, for analytical tractability, the inner, middle1, middle 2 and outer layers will be separated by circumferences of radii $R_{t h}, R_{M_{1}}$ and $R_{M_{2}}$.

In the context of this paper, the ratio between the middle and outer areas are set to 0.2 , as proposed by Wang et al. in [4], which is shown to be a good choice in order to increase fairness among MSs [17]. Accordingly, when using the multi-layer FFR scheme, we have

$$
\frac{R_{M_{1}}^{2}-R_{t h}^{2}}{R^{2}-R_{M_{2}}^{2}}=\frac{R_{M_{2}}^{2}-R_{M_{1}}^{2}}{R^{2}-R_{M_{2}}^{2}}=\frac{1}{5} .
$$

Note that, from (2), $R_{M_{1}}$ and $R_{M_{2}}$ can be written in terms of the distance threshold $R_{t h}$.

Furthermore, the set $\mathcal{F}_{T}$ is split into sets $\mathcal{F}_{I}, \mathcal{F}_{M}$ and $\mathcal{F}_{O}$ of subcarriers allocated to the centre, middle and outer layers, respectively. Sets $\mathcal{F}_{M}$ and $\mathcal{F}_{O}$ are further split into three equal parts, namely $\mathcal{F}_{M 1}, \mathcal{F}_{M 2}$ and $\mathcal{F}_{M 3}$, which are allocated to middle-cell MSs and $\mathcal{F}_{O 1}, \mathcal{F}_{O 2}$ and $\mathcal{F}_{O 3}$ which are allocated to outer-cell MSs, respectively (see Fig. 2). We have that $N_{T}=N_{I}+3 N_{M}+3 N_{O}$, where $N_{M}$ is the number of subcarriers allocated to each of the middle layers.

\section{STATISTICAL CHANNEL CHARACTERIZATION}

The downlink channel is subject to path loss and smallscale fading ${ }^{2}$. The path loss characterising the link between the $b$ th BS and the $u$ th MS can be modeled as

$$
L_{\mathrm{dB}}\left(d_{b, u}\right)=K+10 \alpha \log _{10}\left(d_{b, u}\right),
$$

where $K$ and $\alpha$ are, respectively, a constant and the path loss exponent, and $d_{b, u}$ is the distance (in metres) between the BS $b$ and the MS $u$.

\footnotetext{
${ }^{2}$ In line with the studies in [8], [11], for analytical simplicity, only pathloss and small scale fading are considered in this paper. In future work this will be extended to consider also shadowing as well.
} 
The instantaneous SINR experienced by MS $u$ in the cell of interest on the $n$th subcarrier during the scheduling period $t$ can then be expressed as

$$
\gamma_{u, n}(t)=\frac{P_{s} L\left(d_{0, u}\right)\left|H_{0, u, n}(t)\right|^{2}}{N_{0} \Delta f+I_{u, n}(t)},
$$

where $P_{s}$ is the power allocated per subcarrier, $H_{b, u, n}(t) \sim \mathcal{C N}(0,1)$ is the frequency response resulting from the small-scale fading channel linking the $b$ th BS to MS $u$ on the $n$th subcarrier during scheduling period $t$, $N_{0}$ is the noise power spectral density, and $I_{u, n}(t)$ denotes the interference term that is given by

$$
I_{u, n}(t)=\sum_{b \in \Phi_{n}} P_{s} L\left(d_{b, u}\right)\left|H_{b, u, n}(t)\right|^{2}
$$

with $\Phi_{n}$ representing the set of interfering BSs, which is subcarrier-dependent as the set of interfering BSs depends on which layer subcarrier $n$ belongs to. In fact, for the two-layer FFR scheme we have

$$
\Phi_{n}=\left\{\begin{array}{ll}
\{1,2, \ldots, 18\}, & n \in \mathcal{F}_{I} \\
\{8,10,12,14,16,18\}, & n \in \mathcal{F}_{O_{1}}
\end{array},\right.
$$

and when the multi-layer FFR scheme is used, we have

$\Phi_{n}= \begin{cases}\{1,2, \ldots, 18\}, & n \in \mathcal{F}_{I} \\ \{2,4,6,7,8,10,11,12,14,15,16,18\}, & n \in \mathcal{F}_{M_{1}} \\ \{1,3,5,8,9,10,12,13,14,16,17,18\}, & n \in \mathcal{F}_{M_{2}} \\ \{8,10,12,14,16,18\}, & n \in \mathcal{F}_{O_{1}} .\end{cases}$

Assuming the use of uniform power allocation, the $P_{s}$ can be obtained as

$$
\begin{aligned}
\text { Two-layer FFR: } P_{s} & =\frac{P_{T}}{\left(N_{I}+N_{O}\right)}, \\
\text { Multi-layer FFR: } P_{s} & =\frac{P_{T}}{\left(N_{I}+2 N_{M}+N_{O}\right)},
\end{aligned}
$$

where $P_{T}$ represents the available transmit power at the BS.

As an important notational remark, note that $L\left(d_{b, u}\right)$ can be expressed in terms of the polar coordinates of MS $u$ with respect to BS 0 as $L\left(d_{0, u}, \theta_{0, u}\right)$ and thus, strictly speaking, $\gamma_{u, n}(t)$ is a function of $d_{0, u}$ and $\theta_{0, u}$. Furthermore, it is shown in [18] that the instantaneous SINR in multicell networks barely depends on the polar angle and thus, from this point onwards, the dependence of $\gamma_{u, n}(t)$ on $\theta_{0, u}$ will be omitted.

Since $^{3} h_{b} \triangleq\left|H_{b, u, n}\right|^{2}$ conforms to an exponential distribution with probability density function (PDF) $f_{h_{b}}(x)=$ $e^{-x} u(x)$, where $u(x)$ represents the unit step function, its corresponding cumulative distribution function (CDF) can be obtained as $\mathbb{P}\left\{h_{b} \leq x\right\}=\left(1-e^{-x}\right) u(x)$. Hence, the CDF of the instantaneous SINR $\gamma_{u, n}$ conditioned on the

\footnotetext{
${ }^{3}$ Note that since the channel is assumed to be stationary, from this point onwards the time dependence (i.e., (t)) of all the variables will be dropped unless otherwise stated.
}

set of small-scale fading gains $\boldsymbol{h} \triangleq\left\{h_{b}\right\}_{\forall b \neq 0}$ for a given location of MS $u$, can be derived from (4) as

$$
\begin{aligned}
F_{\gamma_{u, n} \mid \boldsymbol{d}, \boldsymbol{h}}(x \mid d, \boldsymbol{h}) \triangleq \mathbb{P}\left\{\gamma_{u, n} \leq x \mid d_{0, u}, \boldsymbol{h}\right\} \\
=\mathbb{P}\left\{h_{0} \leq \frac{\left(N_{0} \Delta f+I_{u, n}\right)}{\bar{\gamma}_{0}} x \mid d_{0, u}, \boldsymbol{h}\right\} \\
=1-e^{-\frac{x\left(N_{0} \Delta f+I_{u, n}\right)}{\bar{\gamma}_{0}}}, \quad x \geq 0,
\end{aligned}
$$

where $\bar{\gamma}_{0}=P_{n} L(d)$ represents the average received signal. Note that distances in the set $\boldsymbol{d}$ can be written in terms of the distance $d_{0, u}=d$ from the serving BS to MS $u$.

Now, using (9) and averaging over the PDFs of the i.i.d. random variables $\boldsymbol{h}$, the conditional CDF of the instantaneous SINR $\gamma_{u, n}^{A}$ experienced by MS $u$ located at distance $d_{0, u}=d$ from the serving $\mathrm{BS}$ and in the region $A$, can be obtained as

$$
\begin{aligned}
& F_{\gamma_{u, n}^{A} \mid d_{0, u}}(x \mid d) \triangleq \mathbb{P}\left\{\gamma_{u, n}^{A} \leq x \mid d_{0, u}\right\} \\
& =\int_{0}^{\infty} \cdots \int_{0}^{\infty}\left(1-e^{-\frac{x\left(N_{0} \Delta f+I_{u, n}\right)}{\bar{\gamma}_{0}}}\right) \prod_{i \in \Phi_{n}} f_{h_{i}}\left(h_{i}\right) \mathrm{d} h_{i} \\
& =1-e^{-\frac{x N_{0} \Delta f}{\bar{\gamma}_{0}}} \int_{0}^{\infty} \cdots \int_{0}^{\infty} e^{-\frac{x\left(\sum_{\left.i \in \Phi_{n} h_{i} \bar{\gamma}_{i}\right)}\right.}{\bar{\gamma}_{0}}} \prod_{i \in \Phi_{n}} e^{-h_{i}} \mathrm{~d} h_{i} \\
& =1-e^{-\frac{x N_{0} \Delta f}{\bar{\gamma}_{0}}} \prod_{i \in \Phi_{n}} \frac{1}{1+\frac{x \bar{\gamma}_{i}}{\bar{\gamma}_{0}}}, x \geq 0,
\end{aligned}
$$

where $A$ is a token used to represent the cell layers ( where $A$ is a token used to represent the cell layers (or regions) $I, M_{1}, M_{2}$, or $O_{1}, f_{h_{i}}\left(h_{i}\right)$ is the PDF of the variable $h_{i}=\left|H_{i, u, n}\right|^{2}$, and $\bar{\gamma}_{i}=P_{n} L\left(d_{i, u}\right)$ is the average interfering signal from each interfering BS.

\section{THROUGHPUT ANALYSIS}

The average cell throughput (measured in bps) for the downlink of the fractional frequency reuse schemes-aided OFDMA-based cellular network can be expressed as

$$
\bar{\eta}^{T}=\sum_{\forall A} \bar{\eta}^{A},
$$

where $\bar{\eta}^{A}$ is the average throughput in cell layer $A$.

Let us define $M_{0}$ as a positive integer random variable representing the number of MSs in the region served by the tagged BS. As MSs are assumed to be uniformly distributed in entire cell region, the probability that an MS is located in cell layer $A$ is

$$
P_{r}^{A}=\frac{\left(R_{U}^{A}\right)^{2}-\left(R_{L}^{A}\right)^{2}}{R^{2}-R_{0}^{2}},
$$

where $R_{L}^{A}$ and $R_{U}^{A}$ denote the lower and upper radii of the circumferences defining layer $A$. Using these definitions, the average throughput in cell layer $A$ can be expressed as shown in (13) and (14) on top of the next page, for both the two-layer and the multi-layer FFR schemes, respectively, where $\bar{\eta}_{n}^{A}\left(k_{A}\right)$ is the average throughput on the $n$th subcarrier when there are $k_{A}$ MSs in cell layer $A$.

Now, defining $M_{A}$ as a non-negative integer random variable representing the number of MSs in cell region $A$, 
Two-layer FFR scheme: $\bar{\eta}^{A}=\sum_{k=1}^{\infty} \mathbb{P}\left\{M_{0}=k\right\} \sum_{k_{I}=0}^{k}\left(\begin{array}{c}k \\ k_{I}, k-k_{I}\end{array}\right)\left(P_{r}^{I}\right)^{k_{I}}\left(P_{r}^{O_{1}}\right)^{k-k_{I}}\left[N_{A} \bar{\eta}_{n}^{A}\left(k_{A}\right)\right]$.

$$
\text { Multi-layer FFR scheme: } \begin{aligned}
\bar{\eta}^{A} & =\sum_{k=1}^{\infty} \mathbb{P}\left\{M_{0}=k\right\} \sum_{k_{I}=0}^{k} \sum_{k_{M_{1}}=0}^{k-k_{I}} \sum_{k_{M_{2}}=0}^{k-k_{I}-k_{M_{1}}}\left(\begin{array}{c}
k \\
k_{I}, k_{M_{1}}, k_{M_{2}}, k-k_{I}-k_{M_{1}}-k_{M_{2}}
\end{array}\right) \\
& \times\left(P_{r}^{I}\right)^{k_{I}}\left(P_{r}^{M_{1}}\right)^{k_{M_{1}}}\left(P_{r}^{M_{2}}\right)^{k_{M_{2}}}\left(P_{r}^{O_{1}}\right)^{k-k_{I}-k_{M_{1}}-k_{M_{2}}}\left[N_{A} \bar{\eta}_{n}^{A}\left(k_{A}\right)\right] .
\end{aligned}
$$

the average throughput on the $n$th subcarrier allocated to cell region $A$ when $M_{A}=k$, can be obtained as

$$
\begin{aligned}
\bar{\eta}_{n}^{A}(k) & =\Delta f \mathbb{E}_{\gamma_{n}^{A} \mid M_{A}}\left\{\log _{2}\left(1+\gamma_{n}^{A}\right) \mid M_{A}=k\right\} \\
& =\Delta f \log _{2} e \int_{0}^{\infty} \frac{1-F_{\gamma_{n}^{A} \mid M_{A}}(x \mid k)}{1+x} \mathrm{~d} x .
\end{aligned}
$$

In order to obtain tractable mathematical expressions for the CDF $F_{\gamma_{n}^{A} \mid M_{A}}(x \mid k)$ has to be calculated and this depends on the specific scheduling policy applied by the resource allocation algorithm. In the following subsections, this CDF will be obtained for the PF and RR scheduling rules.

\section{A. PF scheduling}

A PF scheduler, exploiting the knowledge of the instantaneous SINRs experienced by all MSs $q \in \mathcal{M}_{A}$, allocates the subcarrier $n \in \mathcal{F}_{A}$ to MS $u \in \mathcal{M}_{A}$ satisfying

$$
u=\arg \max _{q \in \mathcal{M}_{A}}\left\{w_{q}(t) \gamma_{q, n}(t)\right\},
$$

where $\mathcal{M}_{A}$ is the set indexing all MSs in cell region $A$, and $w_{q}(t)=1 / \mu_{q}(t)$ is the weighting (prioritisation) coefficient for MS $q$ that, in this case, depends on the short-term average evolution of channel-state information. This can be obtained using a moving average over a window of $W$ scheduling periods as

$$
\mu_{q}(t)=\left(1-\frac{1}{W}\right) \mu_{q}(t-1)+\sum_{n \in \mathcal{F}_{A}} \iota_{q, n}(t) \frac{\gamma_{q, n}(t)}{W},
$$

with $\iota_{q, n}(t)$ denoting the indicator function of the event that MS $q$ is scheduled to transmit on the $n$th subcarrier during scheduling period $t$, that is,

$$
\iota_{q, n}(t)= \begin{cases}1, & \text { if MS } q \text { is scheduled on carrier } n \text { in } t \\ 0, & \text { otherwise. }\end{cases}
$$

Using this definition, and taking into account that on each subcarrier $n$ in region $A$, and after averaging over the distance to the BS, the MSs are statistically equivalent in terms of SINR for the PF scheduler [19], the conditional $\mathrm{CDF}$ in (15) is given by

$$
F_{\gamma_{n}^{A} \mid M_{A}, \boldsymbol{d}}(x \mid k, \boldsymbol{d})=\frac{1}{k} \sum_{u \in \mathcal{M}_{A}} F_{\gamma_{u, n}^{A} \mid d_{0, u}}^{k}\left(x \mid d_{0, u}\right) \text {. }
$$

Now, taking into account that on each subcarrier $n$ in region $A$, and after averaging over the distance to the
BS, the MSs are statistically equivalent in terms of SINR, the (unconditional) random variables $\left\{\gamma_{q, n}(t)\right\}_{\forall q \in \mathcal{M}_{A}}$ are i.i.d., and the conditional CDF in (15) can be obtained as

$$
F_{\gamma_{n}^{A} \mid M_{A}}^{\mathrm{PF}}(x \mid k)=\int_{R_{L}^{A}}^{R_{U}^{A}} F_{\gamma_{u, n}^{A} \mid d_{0, u}}^{k}(x \mid d) f_{d_{0, u}}(d) \mathrm{d} d,
$$

where $f_{d_{0, u}}(d)$ is the PDF of the random variable $d_{0, u}$ that can be expressed as

$$
f_{d_{0, u}}(d)=\frac{2 d}{R_{U}^{A^{2}}-R_{L}^{A^{2}}}, \quad R_{L}^{A} \leq d \leq R_{U}^{A} .
$$

Using (21), (20) and (15) in (13) or (14) and after some algebraic manipulations, the average throughput in cell layer $A$, for the PF scheduling rule, can be obtained as shown in (22) on top of the next page.

In order to analyze the capacity achieved by the worst $\mathrm{MSs}^{4}$ of each layer, we define the edge of region $A$ as a thin angular region with lower radius $R_{L}^{A \text {,edge }}=$ $R_{U}^{A}-\delta$ and upper radius $R_{U}^{A \text {,edge }}=R_{U}^{A}$, where $\delta \leq$ $\left(R_{U}^{A}-R_{L}^{A}\right) / 2$.

\section{B. RR scheduling}

A RR scheduler allocates subcarriers to MSs in a fair time-sharing approach. Since the SINRs experienced by MSs in region $A$ on each subcarrier $n$ are statistically equivalent, serving $M_{A}=k$ MSs using a RR scheduling policy is equivalent to serving $M_{A}=1 \mathrm{MS}$ with $\mathrm{PF}$ (even when MSs are selected with non uniform probability). Therefore, the conditional CDF in (15) simplifies to

$$
F_{\gamma_{n}^{A}}^{\mathrm{RR}}(x)=F_{\gamma_{n}^{A} \mid M_{A}}^{\mathrm{PF}}(x \mid 1) .
$$

Finally, using (23), (21), and (15) in (13) or (14) and after some algebraic manipulations, the average throughput in the cell layer $A$, for the RR scheduling rule, can be obtained as shown in (24) on top of the next page.

\section{PERFORMANCE EVALUATION}

In order to validate the proposed analytical framework, a 19-cell network is considered, where the cell of interest is surrounded by two rings of interfering BSs (see Figs. 1 and 2). As stated in previous sections, MSs are distributed over the coverage area using a PPP of normalized intensity $\lambda$ (measured in MSs per area unit). For the sake of

${ }^{4}$ When the shadowing is not taken into consideration, the worst MSs are located in the edge region of each layer. 
$\mathrm{PF}: \bar{\eta}^{A}=\frac{2 \log _{2} e N_{A} \Delta f}{R_{U}^{A^{2}}-R_{L}^{A^{2}}} \int_{0}^{\infty} \int_{R_{L}^{A}}^{R_{U}^{A}}\left(1-\exp \left[-\pi \lambda\left(R^{2}-R_{0}^{2}\right) P_{r}^{A}\left(1-F_{\gamma_{u, n}^{A} \mid d_{0, u}}(x \mid d)\right)\right]\right) \frac{d}{1+x} \mathrm{~d} d \mathrm{~d} x$.

$\mathrm{RR}: \bar{\eta}^{A}=\frac{2 \log _{2} e N_{A} \Delta f}{R_{U}^{A^{2}}-R_{L}^{A^{2}}}\left(1-\exp \left[-\pi \lambda\left(R^{2}-R_{0}^{2}\right) P_{r}^{A}\right]\right) \int_{0}^{\infty} \int_{R_{L}^{A}}^{R_{U}^{A}}\left(1-F_{\gamma_{u, n}^{A} \mid d_{0, u}}(x \mid d)\right) \frac{d}{1+x} \mathrm{~d} d \mathrm{~d} x$.

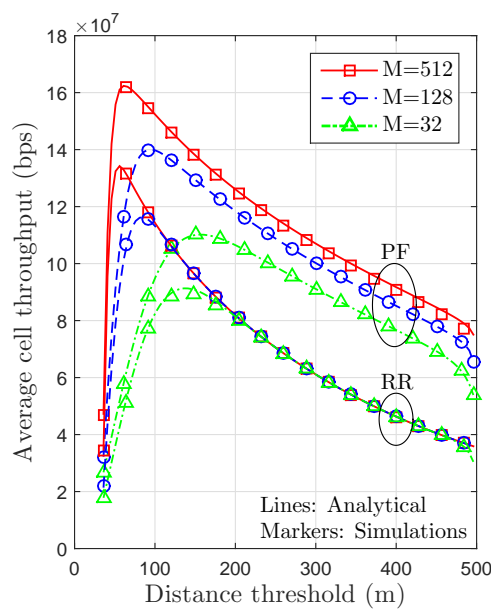

(a) Total cell throughput

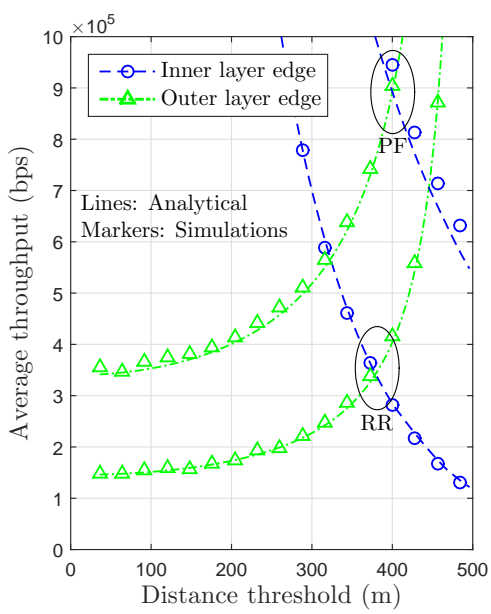

(b) Layer's edge throughput, $M=512$

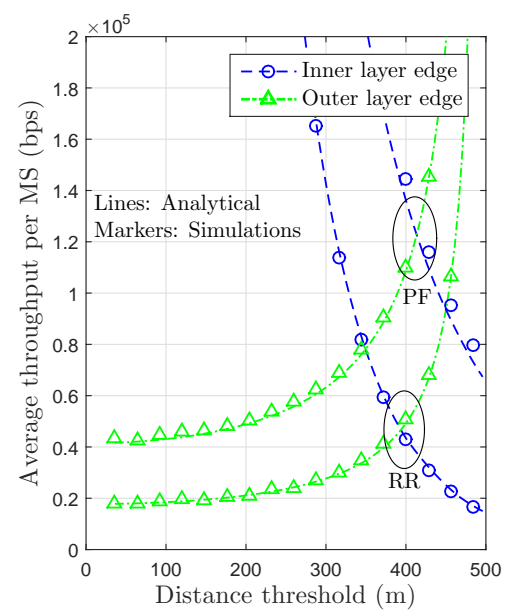

(c) Layer's edge throughput per MS

Fig. 3: Average cell throughput, layer's edge throughput and layer's edge throughput per MS, for both RR and PF scheduling policies (two-layer FFR).

Table I: Network parameters

\begin{tabular}{|c|c|}
\hline \hline System parameter & Value \\
\hline Cell radius & $500 \mathrm{~m}$ \\
Minimum distance between BS and MSs & $35 \mathrm{~m}$ \\
Distance $\delta$ defining the layer edge & $4 \mathrm{~m}$ \\
Transmit power at the BS & $46 \mathrm{dBm}$ \\
Antenna gain at the BS & $14 \mathrm{dBi}$ \\
Noise power spectral density & $-174 \mathrm{dBm} / \mathrm{Hz}$ \\
Receiver noise figure & $7 \mathrm{~dB}$ \\
Total bandwidth & $20 \mathrm{MHz}$ \\
Subcarrier spacing & $15 \mathrm{kHz}$ \\
Occupied subcarriers (including DC) & 1201 \\
Number of inner subcarriers & 624 \\
Number of middle subcarriers & 32 \\
Path loss model (dB) & $15.3+37.6 \log _{10}(d)$ \\
Monte Carlo trials & 1,000 \\
\hline \hline
\end{tabular}

presentation clarity, results in this section will be shown as a function of the average number of MSs per cell $\left(M \triangleq \pi \lambda\left(R^{2}-R_{0}^{2}\right)\right)$ The main system parameters used to generate both the analytical and simulation results are based on [20] and are summarized in Table I.

Illustrating the system behaviour, results in Figure 3 are provided applying the two-layer FFR scheme and using both PF and RR scheduling policies. For the sake of clarity, lines are used to represent the analytical results and markers correspond to Monte-Carlo simulations. It is worth noting the very good agreement between the simulated and analytical results, thus validating the novel mathematical framework.
Focusing now on performance aspects, Fig. 3a presents the average throughput, considering the whole coverage cell, as a function of the distance threshold $R_{t h}$. As expected, PF outperforms RR because PF is a channelaware scheduler exploiting the multiuser diversity. The maximum average cell throughput increases with the average number of MSs per cell. This is basically due to two distinct effects. The first one, only exploited by the $\mathrm{PF}$ scheduling rule, is caused by the degree of multiuser diversity provided by the increase of $M$. The second effect, affecting all the schedulers but more noticeable when using the RR scheduler, is because increasing the average number of MSs per cell raises the probabilities of having at least one inner MS and one outer MS, hence reducing the probability of waste of resources.

Figure $3 b$ presents the average throughput of the worst MSs typically located at each layer's edge, as a function of the distance threshold $R_{t h}$. Note that, as the inner layer becomes larger the throughput of the inner layer's edge decreases because the MSs located in the edge of the inner layer suffer from high levels of ICI, whereas the throughput of outer layer increases. This phenomenon is consistent with the results shown in Fig. 3c. In order to analyze the average capacity achieved by an arbitrary MS located in the edge of certain layer, this figure presets the layer's edge throughput per MS as a function of the distance threshold $R_{t h}$. It is interesting to note that the worst MSs are not necessarily located in the outer layer. 


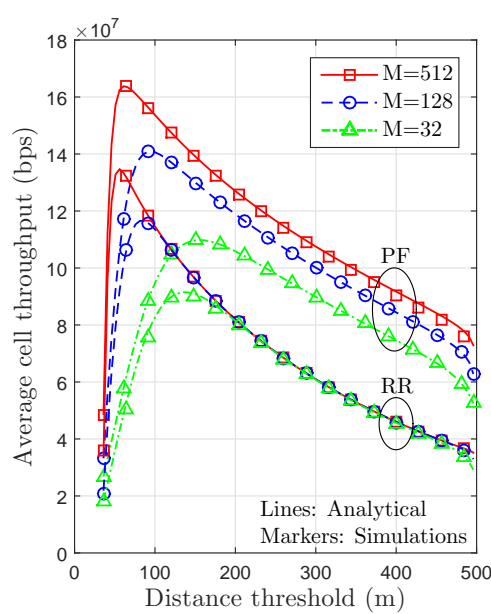

(a) Total cell throughput

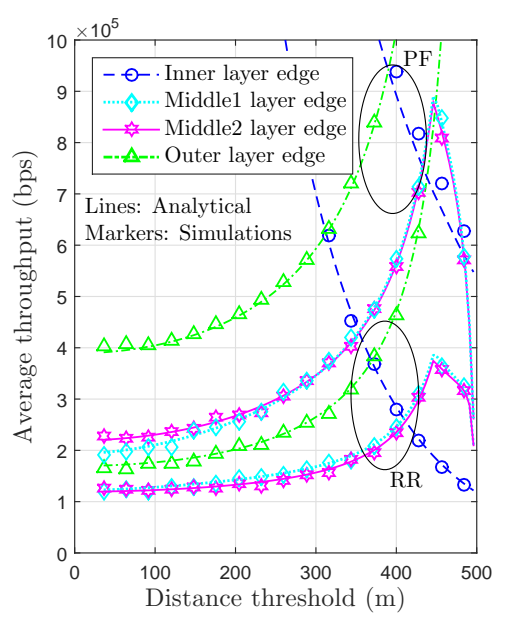

(b) Layer's edge throughput, $M=512$

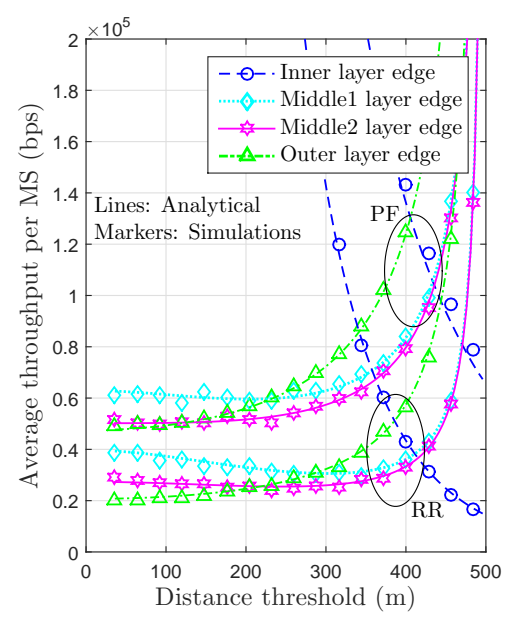

(c) Layer's edge throughput per MS

Fig. 4: Average cell throughput, layer's edge throughput and layer's edge throughput per MS, for both RR and PF scheduling policies (multi-layer FFR).

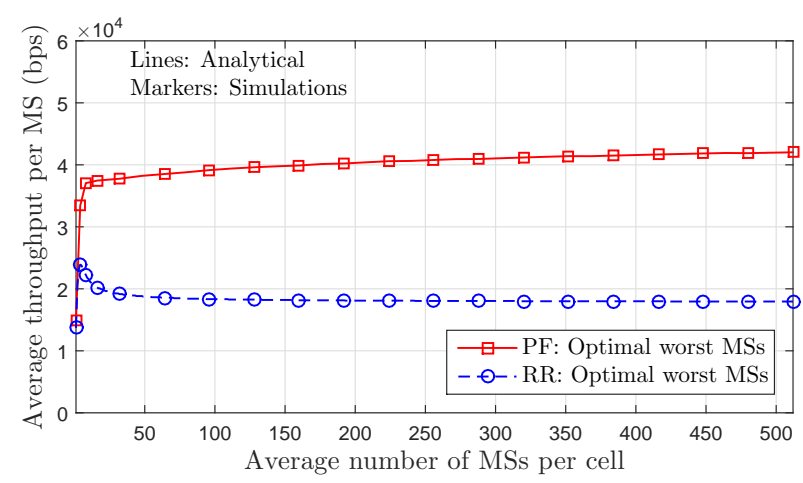

(a) Two-layer FFR

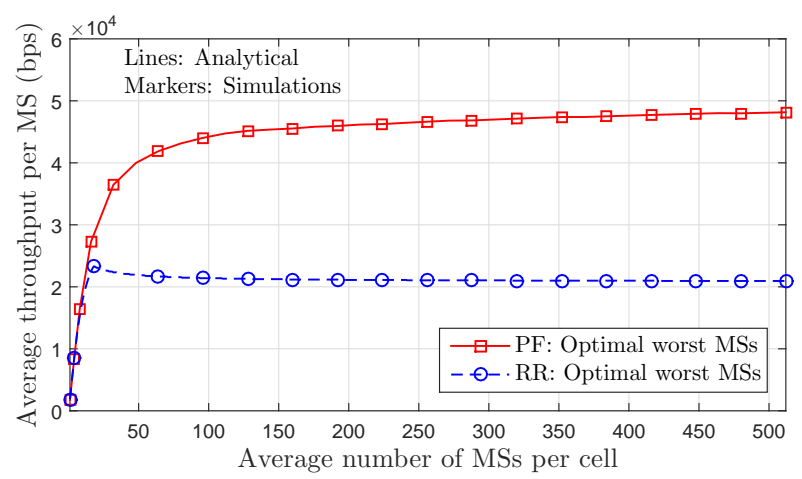

(b) Multi-layer FFR

Fig. 5: Optimal layer's edge throughput, for both RR and PF scheduling policies, and under both two-layer and multi-layer schemes).

Figure 4 shows the performance of the multi-layer scheme, the same trend is observed when comparing both scheduling policies (see Fig. 3). Checking the maximum average cell throughput in Fig. 4a, notice that the multilayer FFR has only slightly improved the whole cell performance compared with the two-layer scheme, due to the increment of the spectrum utilization when the multilayer scheme is used. Regardless of the scheduling policy in use, the middle 1 layer performance is always better than the middle2 layer performance due to the fact that both of them hold the same reuse factor while the middle1 layer is nearer to the corresponding BS (see Figs. $4 \mathrm{~b}$ and $4 \mathrm{c}$ ). It is also interesting to note in Fig. 4c that, for a low value of $R_{t h}$, the worst MSs are located in the edge of the outer layer, however, as $R_{t h}$ increases, the MSs located in the edge of the middle 2 or the inner layer become the worst.

The optimization outcomes are shown in Fig. 5 when using both $\mathrm{PF}$ and RR scheduling policies, and under both two-layer and multi-layer schemes. Regardless of the scheme in use, for each maximum value of average cell throughput, the corresponding optimal value of the worst
MSs' capacity increases little with $M$ when using PF (e.g. for $M=32$ under the two-layer FFR this value is equal to $37.76 \mathrm{Kbps}$, whereas for $M=512$ this value is equal to $42.02 \mathrm{Kbps}$ ). In contrast, the corresponding optimal value of the worst MSs' capacity decreases little with $M$ when using RR (e.g. for $M=32$ under the two-layer FFR this value is equal to $19.14 \mathrm{Kbps}$, whereas for $M=512$ this value is equal to $17.95 \mathrm{Kbps}$ ). The main advantage of the multi-layer scheme is that, without any sacrifice in spectral efficiency, it is able to provide higher levels of fairness between the MSs located accros the coverage area of the network, a QoS metric of paramount importance in beyond-4G cellular networks. In particular, for the multilayer FFR, it can be observed that the average throughput for the worst MSs when $M=512$ is equal to 48.16 Kbps when using PF and equal to $20.94 \mathrm{Kbps}$ when using $\mathrm{RR}$, corresponding to a $14.6 \%$ and $16.7 \%$ improvement, respectively, compared to the benchmark two-layer FFR scheme. 
García-Morales, Femenias, Riera-Palou, 2017.

\section{CONCLUSION}

This paper has presented and validated a novel analytical framework to evaluate the performance of the multi-layer FFR scheme in a downlink OFDMA-based cellular network. Mathematically tractable solutions have been derived for popular scheduling rules, namely, PF and RR. For the specific case of PF scheduling, the cell throughput improvement is further accentuated by a greater exploitation of the multiuser diversity. Remarkably, as the average number of MSs per cell increases, the maximum average cell throughput also increases whereas the optimal value of the worst MSs' capacity remains virtually constant. Results show that the multi-layer FFR scheme does not decrease the cell spectral efficiency or the average throughput of the whole cell while significantly increases the worst MSs' capacity throughout the cell at the cost of sacrificing the throughput of MSs located close to the BS. In other words, the multi-layer FFR scheme leads to an overall cell capacity virtually identical to that of two-layer FFR, but it is able to archive a higher degree of fairness. Further work will concentrate on the use of more sophisticated ICIC techniques (e.g., soft/adaptive frequency reuse schemes, higher order sectorization, network MIMO).

\section{ACKNOWLEDGMENTS}

Work supported by the Agencia Estatal de Investigación and Fondo Europeo de Desarrollo Regional (AEI/FEDER, UE) under project ELISA (subproject TEC2014-59255C3-2-R), Ministerio de Economía y Competitividad (MINECO), Spain, and the Conselleria d'Educació, Cultura i Universitats (Govern de les Illes Balears) under grant FPI/1538/2013 (co-financed by the European Social Fund). The research leading to these results has also received funding from "la Caixa" Banking Foundation.

\section{REFERENCES}

[1] E. Dahlman, S. Parkvall, and J. Skold, 4G: LTE/LTE-Advanced for Mobile Broadband, 2nd ed. Elsevier Science, 2013.

[2] A. S. Hamza, S. S. Khalifa, H. S. Hamza, and K. Elsayed, "A survey on inter-cell interference coordination techniques in OFDMA-based cellular networks," IEEE Communications Surveys \& Tutorials, vol. 15 , no. 4, pp. 1642-1670, 2013.

[3] N. Saquib, E. Hossain, and D. I. Kim, "Fractional frequency reuse for interference management in LTE-Advanced HetNets," IEEE Wireless Communications, vol. 20, no. 2, pp. 113-122, 2013.

[4] L. Wang, F. Fang, N. Nikaein, and L. Cottatellucci, "An analytical framework for multilayer partial frequency reuse scheme design in mobile communication systems," IEEE Transactions on Vehicular Technology, vol. 65, no. 9, pp. 7593-7605, 2016.

[5] F. Kelly, A. Maulloo, and D. Tan, "Rate control for communication networks: shadow prices, proportional fairness and stability," The Journal of the Operational Research Society, vol. 49, no. 3, pp. 237-252, 1998.

[6] T. Novlan, R. Ganti, A. Ghosh, and J. Andrews, "Analytical evaluation of fractional frequency reuse for OFDMA cellular networks," IEEE Transactions on Wireless Communications, vol. 10, no. 12, pp. 4294-4305, December 2011.

[7] H. ElSawy, E. Hossain, and M. Haenggi, "Stochastic geometry for modeling, analysis, and design of multi-tier and cognitive cellular wireless networks: A survey," IEEE Communications Surveys \& Tutorials, vol. 15, no. 3, pp. 996-1019, 2013.

[8] F. Jin, R. Zhang, and L. Hanzo, "Fractional frequency reuse aided twin-layer femtocell networks: Analysis, design and optimization,' IEEE Transactions on Communications, vol. 61, no. 5, pp. 2074 2085, 2013.
[9] M. Assaad, "Optimal fractional frequency reuse (FFR) in multicellular OFDMA system," in IEEE 68th Vehicular Technology Conference (VTC-Fall), 2008, pp. 1-5.

[10] A. Najjar, N. Hamdi, and A. Bouallegue, "Efficient frequency reuse scheme for multi-cell OFDMA systems," in IEEE Symposium on Computers and Communications (ISCC), 2009, pp. 261-265.

[11] Z. Xu, G. Y. Li, C. Yang, and X. Zhu, "Throughput and optimal threshold for FFR schemes in OFDMA cellular networks," IEEE Transactions on Wireless Communications, vol. 11, no. 8, pp. 27762785, 2012.

[12] J. García-Morales, G. Femenias, and F. Riera-Palou, "Analytical performance evaluation of OFDMA-based heterogeneous cellular networks using FFR," in IEEE 81st Vehicular Technology Conference (VTC-Spring), 2015.

[13] G. Femenias and F. Riera-Palou, "Corrections to, and comments on, "Throughput and Optimal Threshold for FFR Schemes in OFDMA Cellular Networks"," IEEE Transactions on Wireless Communications, vol. 14, no. 5, pp. 2926 - 2928, 2015.

[14] Z. Xie and B. Walke, "Performance analysis of reuse partitioning techniques in ofdma based cellular radio networks," in Telecommunications (ICT), 2010 IEEE 17th International Conference on IEEE, 2010, pp. 272-279.

[15] R. Ghaffar and R. Knopp, "Fractional frequency reuse and interference suppression for ofdma networks," in Modeling and Optimization in Mobile, Ad Hoc and Wireless Networks (WiOpt), 2010 Proceedings of the 8th International Symposium on. IEEE, 2010, pp. 273-277.

[16] X. Yang, "A multilevel soft frequency reuse technique for wireless communication systems," IEEE Communications Letters, vol. 18, no. 11, pp. 1983-1986, 2014.

[17] L. Wang, F. Fang, K. Min, N. Nikaein, and L. Cottatellucci, "Toward multi-layer partial frequency reuse in future mobile communication systems," in Communications in China (ICCC), 2014 IEEE/CIC International Conference on. IEEE, 2014, pp. 647-652.

[18] M. Maqbool, P. Godlewski, M. Coupechoux, and J.-M. Kélif, "Analytical performance evaluation of various frequency reuse and scheduling schemes in cellular OFDMA networks," Performance Evaluation, vol. 67, no. 4, pp. 318-337, 2010

[19] J. García-Morales, G. Femenias, and F. Riera-Palou, "On the analysis of channel-aware schedulers in OFDMA-based networks using FFR," in 11th International Conference on Wireless and Mobile Computing, Networking and Communications (WiMob). IEEE, 2015, pp. 786-793.

[20] G. TR36.921, "Home enode b (HeNB) radio frequency (RF) requirements analysis (release 9)," v9.0.0. Mar. 2010. 